\title{
Intraoperative three-dimensional versus two-dimensional echocardiography for left ventricular assessment
}

\author{
Meris, Alessandra ; Santambrogio, Luisa ; Casso, Gabriele ; Mauri, Romano ; Engeler, Albin ; Cassina, \\ Tiziano
}

\begin{abstract}
BACKGROUND Three-dimensional (3D) transesophageal echocardiography (TEE) technology is now widely used intraoperatively in cardiac surgery. Left ventricular (LV) measurements with 3D transthoracic echocardiography correlate better with cardiac magnetic resonance measurements compared with traditional two-dimensional (2D) transthoracic echocardiography. In this study, we compared intraoperative 3D TEE against 2D TEE regarding quantitative indices of LV function. METHODS We performed 2D TEE and 3D TEE examinations on 156 patients scheduled for elective cardiac surgery. Two-dimensional TEE images of midesophageal 4-, 2-chamber, and long-axis views were acquired. LV volumes and ejection fraction (EF) were calculated by Simpson's method. Three-dimensional full-volume images were recorded to calculate by a semiautomated procedure LV volumes (indexed to body surface area) and EF. 3D and 2D LV dimensions and function, image quality, time for acquisition/analyses, and reproducibility were compared by the Wilcoxon matched-pairs signed-ranks test. Pairwise differences between 3D and 2D data were compared using 95\% prediction intervals (PIs) and Bland-Altman methodology. 3D volumes were also plotted against $2 \mathrm{D}$ volumes in scatter plots using a 3-zone error grid. RESULTS There was no significant difference between $3 \mathrm{D}$ and $2 \mathrm{D}$ in the estimation of $\mathrm{EF}(\mathrm{P}=$ 0.227 ; median pairwise difference, $-0.4 \%$ [95\% PIs, $-8.6 \%$ to $8.8 \%]$ ). 3D LV indexed end-diastolic volumes (iEDVs) and end-systolic volumes (iESVs) were larger than $2 \mathrm{D}$ iEDVs $(\mathrm{P}<0.001$; median pairwise difference, $3.3 \mathrm{~mL} / \mathrm{m}$ [95\% PIs, -9.4 to $14.1 \mathrm{~mL} / \mathrm{m}$ ] and iESV: $\mathrm{P}<0.001$; median pairwise difference, 1.4 $\mathrm{mL} / \mathrm{m}$ [95\% PIs, -5.2 to $10.1 \mathrm{~mL} / \mathrm{m}])$. In the vast majority of cases $(98.8 \%$ of cases for iEDV and $92.8 \%$ of cases for iESV), the difference between 2D and 3D TEE indexed volumes did not alter classification into normal, mildly to moderately dilated, or severely dilated volumes, as demonstrated by the 3-zone error grid analysis. Acquisition of 3D TEE image and analysis were not feasible in 4 patients $(2.5 \%)$ for whom a quantitative 2D assessment of the LV was also impossible. 3D and 2D quality image was similar $(\mathrm{P}=0.206)$. There was no difference in $3 \mathrm{D}$ versus $2 \mathrm{D}$ acquisition time $(\mathrm{P}=0.805$; pairwise difference $=2$ seconds [95\% PIs, -20 to 35 seconds] $)$, but 3D analysis required more time $(\mathrm{P}<0.001$; pairwise difference $=117$ seconds [95\% PIs, 66 to 197 seconds]). Differences in repeated 3D versus 2D indexed volumes were not statistically significant, both considering interobserver reproducibility (iEDV: $\mathrm{P}=0.125$; pairwise difference, $0.26 \pm 1.76 \mathrm{~mL}[95 \% \mathrm{PIs},-3.58$ to $3.73 \mathrm{~mL}$ ] and $\mathrm{iESV}: \mathrm{P}=0.126$; pairwise difference, -0.16 $\pm 1.67 \mathrm{~mL}[95 \%$ PIs, -3.96 to $3.69 \mathrm{~mL}]$ ) and intraobserver reproducibility (iEDV: $\mathrm{P}=0.975$; pairwise difference, $-0.02 \pm 1.20 \mathrm{~mL}$ [95\% PIs, -2.32 to $2.08 \mathrm{~mL}$ ] and iESV: $\mathrm{P}=0.228$; pairwise difference, -0.19 $\pm 1.13 \mathrm{~mL}$ [95\% PIs, -2.47 to $2.53 \mathrm{~mL}]$ ). CONCLUSIONS Intraoperative 3D TEE quantification of LV global function, image acquisition time, and reproducibility was not statistically different when compared with 2D TEE. It was however associated with calculation of larger LV volumes and a longer analysis time. Nevertheless, the 3-zone error grid analysis of the LV indexed volumes showed that the difference between $3 \mathrm{D}$ and 2D measurements does not affect the LV classification as normal, mildly to moderately dilated, or severely dilated.
\end{abstract}


Posted at the Zurich Open Repository and Archive, University of Zurich

ZORA URL: https://doi.org/10.5167/uzh-109585

Journal Article

Published Version

Originally published at:

Meris, Alessandra; Santambrogio, Luisa; Casso, Gabriele; Mauri, Romano; Engeler, Albin; Cassina, Tiziano (2014). Intraoperative three-dimensional versus two-dimensional echocardiography for left ventricular assessment. Anesthesia and Analgesia, 118(4):711-720.

DOI: https://doi.org/10.1213/ANE.0000000000000093 


\title{
Intraoperative Three-Dimensional Versus Two-Dimensional Echocardiography for Left Ventricular Assessment
}

\author{
Alessandra Meris, MD, Luisa Santambrogio, MD, Gabriele Casso, MD, Romano Mauri, MD, \\ Albin Engeler, MD, and Tiziano Cassina, MD
}

\begin{abstract}
BACKGROUND: Three-dimensional (3D) transesophageal echocardiography (TEE) technology is now widely used intraoperatively in cardiac surgery. Left ventricular (LV) measurements with 3D transthoracic echocardiography correlate better with cardiac magnetic resonance measurements compared with traditional two-dimensional (2D) transthoracic echocardiography. In this study, we compared intraoperative 3D TEE against 2D TEE regarding quantitative indices of LV function.

METHODS: We performed 2D TEE and 3D TEE examinations on 156 patients scheduled for elective cardiac surgery. Two-dimensional TEE images of midesophageal 4-, 2-chamber, and long-axis views were acquired. LV volumes and ejection fraction (EF) were calculated by Simpson's method. Three-dimensional full-volume images were recorded to calculate by a semiautomated procedure LV volumes (indexed to body surface area) and EF. 3D and 2D LV dimensions and function, image quality, time for acquisition/analyses, and reproducibility were compared by the Wilcoxon matchedpairs signed-ranks test. Pairwise differences between 3D and 2D data were compared using 95\% prediction intervals (PIs) and Bland-Altman methodology. 3D volumes were also plotted against 2D volumes in scatter plots using a 3-zone error grid.

RESULTS: There was no significant difference between $3 \mathrm{D}$ and $2 \mathrm{D}$ in the estimation of EF $(P=0.227$; median pairwise difference, $-0.4 \%$ [95\% PIs, $-8.6 \%$ to $8.8 \%])$. 3D LV indexed end-diastolic volumes (iEDVs) and end-systolic volumes (iESVs) were larger than 2D iEDVs ( $P$ $<0.001$; median pairwise difference, $3.3 \mathrm{~mL} / \mathrm{m}^{2}$ [95\% PIs, -9.4 to $14.1 \mathrm{~mL} / \mathrm{m}^{2}$ ] and $\mathrm{iESV}$ : $P<0.001$; median pairwise difference, $1.4 \mathrm{~mL} / \mathrm{m}^{2}$ [95\% Pls, -5.2 to $\left.10.1 \mathrm{~mL} / \mathrm{m}^{2}\right]$ ). In the vast majority of cases (98.8\% of cases for iEDV and $92.8 \%$ of cases for iESV), the difference between $2 \mathrm{D}$ and 3D TEE indexed volumes did not alter classification into normal, mildly to moderately dilated, or severely dilated volumes, as demonstrated by the 3-zone error grid analysis. Acquisition of 3D TEE image and analysis were not feasible in 4 patients $(2.5 \%)$ for whom a quantitative $2 \mathrm{D}$ assessment of the LV was also impossible. 3D and 2D quality image was similar $(P=0.206)$. There was no difference in 3D versus $2 \mathrm{D}$ acquisition time $(P=0.805$; pairwise difference $=2$ seconds [95\% Pls, -20 to 35 seconds]), but 3D analysis required more time $(P<0.001$; pairwise difference $=117$ seconds $[95 \%$ Pls, 66 to 197 seconds] $)$. Differences in repeated $3 \mathrm{D}$ versus $2 \mathrm{D}$ indexed volumes were not statistically significant, both considering interobserver reproducibility (iEDV: $P=0.125$; pairwise difference, $0.26 \pm 1.76 \mathrm{~mL}[95 \% \mathrm{Pls}$, -3.58 to $3.73 \mathrm{~mL}$ ] and iESV: $P=0.126$; pairwise difference, $-0.16 \pm 1.67 \mathrm{~mL}[95 \%$ Pls, -3.96 to $3.69 \mathrm{~mL}$ ) and intraobserver reproducibility (iEDV: $P=0.975$; pairwise difference, $-0.02 \pm$ $1.20 \mathrm{~mL}$ [95\% PIs, -2.32 to $2.08 \mathrm{~mL}$ ] and $\mathrm{ESSV}: P=0.228$; pairwise difference, $-0.19 \pm 1.13$ $\mathrm{mL}$ [95\% Pls, -2.47 to $2.53 \mathrm{~mL}]$ ).

CONCLUSIONS: Intraoperative 3D TEE quantification of LV global function, image acquisition time, and reproducibility was not statistically different when compared with 2D TEE. It was however associated with calculation of larger LV volumes and a longer analysis time. Nevertheless, the 3-zone error grid analysis of the LV indexed volumes showed that the difference between 3D and 2D measurements does not affect the LV classification as normal, mildly to moderately dilated, or severely dilated. (Anesth Analg 2014;118:711-20)
\end{abstract}

From the Division of Cardiothoracic Anesthesiology, Fondazione Cardiocentro Ticino, Lugano, Switzerland.

Accepted for publication November 26, 2013.

Funding: Not funded.

The authors declare no conflicts of interest.

Reprints will not be available from the authors.

Copyright $\odot 2014$ International Anesthesia Research Society

DOI: 10.1213 /ANE.0000000000000093
Tntraoperative transesophageal echocardiography (TEE) is now a well-established monitoring technique during cardiac surgery. TEE allows assessment of changes in left ventricular (LV) preload and afterload and for evaluation of myocardial dysfunction. ${ }^{1-3}$

Address correspondence to Alessandra Meris, MD, Division of Cardiothoracic Anesthesiology, Fondazione Cardiocentro Ticino, Via Tesserete 48, 6900 Lugano, Switzerland. Address e-mail to a.meris@hotmail.com. 
Nevertheless, the reproducibility and accuracy of twodimensional (2D) echocardiography may be limited mainly because the LV volume measurements are based on geometric assumptions and the LV apex is often foreshortened, particularly with TEE. ${ }^{4,5}$ In patients with cardiomyopathies, the preconceived assumption of a normal LV shape used in calculation could lead to mistakes in quantifying volumes., ${ }^{4,6}$ In the past, three-dimensional (3D) echocardiography was cumbersome and time consuming, limiting its application to research or studies outside the operating room. Recent technical improvements have allowed real-time assessments with faster and easier use of 3D echocardiography. ${ }^{7,8}$ In this setting, several studies using transthoracic echocardiography (TTE) have definitely demonstrated the superiority of 3D over 2D echocardiography, because semiautomated detection of the LV endocardial borders allows rapid, accurate, and reproducible measurements of LV volumes, showing higher correlation with the reference standards of magnetic resonance imaging (MRI) and ventriculography, ${ }^{4,9-11}$ 3D echocardiography eliminates the need for cognitive reconstruction of LV shape by the clinician operator and use of geometric assumptions for LV quantification. So far, however, there is no evidence showing the feasibility and the potential advantages of intraoperative 3D LV evaluation in comparison to 2D TEE traditional procedures. ${ }^{12-14}$

The aim of this study was to evaluate the intraoperative use of 3D TEE compared with 2D TEE for assessment of LV volumes in patients undergoing elective cardiac surgery. Specifically, we compared the following: (1) LV volumes and ejection fraction (EF) quantified by 3D and 2D TEE; (2) the $3 \mathrm{D}$ and $2 \mathrm{D}$ image quality and time requirement for acquisition and analysis; and (3) the 3D and 2D intra- and interobserver reproducibility for quantification of LV volumes.

\section{METHODS}

\section{Study Population}

Between September 2009 and December 2010, we prospectively enrolled 156 consecutive patients who underwent elective cardiac surgery. Exclusion criteria are listed in Table 1 . In addition, 4 study patients were excluded for inadequate endocardial visualization, and thus, the final

\begin{tabular}{lc} 
Table 1. Exclusion Criteria & No. of patients \\
Exclusion criteria & 39 \\
Rhythm other than sinus & 13 \\
Congenital cardiac disease & 50 \\
Hemodynamically unstable condition requiring IV & \\
$\quad$ catecholamines or IABP before cardiac surgery & 12 \\
Extracorporeal membrane oxygenation & 9 \\
Esophageal pathologies contraindicating TEE & 67 \\
Not elective cardiac surgery & \\
\hline
\end{tabular}

$\mathrm{IABP}=$ intraaortic balloon pump; TEE = transesophageal echocardiography cohort consisted of 152 patients. All subjects gave written informed consent, and the study was approved by the IRB of Cardiocentro Hospital in Lugano, Switzerland.

\section{Echocardiography}

TEE images of the LV were acquired using an iE33 ultrasound system (Philips Medical Systems, Andover, MA) equipped with a fully sampled 3D matrix array transducer (X7-2t). Both 2D and 3D images were acquired after induction of general anesthesia before sternotomy, with the patient in supine position, under hemodynamically stable conditions, pausing mechanical ventilation in the end-expiratory phase. 2D and 3D images were acquired within several heartbeats by the same experienced operator (AM) who also performed all the analyses. The anesthetic was managed in collaboration with a qualified anesthesia nurse according to our routine institutional practice.

Acquisition time started with the first attempt to find an adequate image with the probe at the midesophageal (ME) level and ending with the recording of that image. Analysis time started with control of all the 2D and 3D images for artifacts. The endocardial definition was assessed for each of the 17-segment models described in the guidelines throughout the cardiac cycle. The image quality was then graded using a 4-point scale, as shown in Table $2 .{ }^{15}, 16$ Analysis time ended with the quantification of $\mathrm{LV}$ volumes and EF.

The papillary muscles and trabeculae were traced at their base and were included in the calculation of the LV volume. End diastole was defined as the frame after mitral valve closure or the frame in the cardiac cycle in which the cardiac dimension is the largest. End systole was identified as the frame preceding mitral valve opening or the frame in the cardiac cycle in which the cardiac dimension is the smallest, according to the current guidelines. ${ }^{17}$

\section{Two-Dimensional Echocardiography}

The 4- and 2-chamber and long-axis views were acquired at the ME level over 3 beats. Every effort was made to minimize foreshortening of the LV cavity.

All the images were analyzed with the Philips iE33 Cardiac Analysis Package (Philips Medical Systems). Two basal points at the level of mitral valve annulus and 1 apical point were identified, and the endocardial borders were automatically defined and traced; they were manually traced by the operator both in 4- and 2-chamber views, as needed. LV volumes and EF were measured online by biplane Simpson's method according to current guidelines. ${ }^{17} 2 \mathrm{D}$ measurements were obtained over 3 separate cardiac cycles and averaged.

\section{Table 2. Two-Dimensional (2D) and 3D Image Quality Assessment with a 4-Point Scale}

\section{Category}

Optimal

Good

Fair

Sufficient
Complete endocardial definition

Inadequate visualization of 1 segment

Inadequate visualization of 2 segments, but adequate visualization of adjacent segments

Inadequate visualization of 3 segments, but adequate visualization of adjacent segments

\begin{tabular}{cc}
\multicolumn{2}{c}{ No. of patients (\%) } \\
\hline 3D & 2D \\
$79(52)$ & $79(52)$ \\
$51(33)$ & $54(36)$ \\
$18(12)$ & $16(10)$ \\
$4(3)$ & $3(2)$
\end{tabular}




\section{Three-Dimensional Echocardiography}

The full-volume 3D echocardiography data set of the LV was acquired starting from the ME 4-chamber view over 4 cardiac cycles and was gated to a stable electrocardiograph recording. To optimize the $3 \mathrm{D}$ image frame rate, the lateral sector width was minimized to include only the mitral and aortic valves so that the minimal frame rate was always $>20$ $\mathrm{Hz}$ (in average $25 \mathrm{~Hz}$ ).

LV analysis was performed online in the operating room using the Philips Q-lab 3D-Advanced quantification software (Philips Medical Systems). ${ }^{18} 4$ - and 2-chamber and transgastric views were automatically extracted from the pyramidal data set, and the quality of endocardial definition was scrutinized by manually rotating the image on its own axis. ${ }^{15}$

Before initiation of the advanced LV quantification process, the ME 4-chamber view was manually adjusted by rotating the images on its long axis to remove the LV outflow tract. At end diastole and end systole, 5 anatomic landmarks were manually identified at the level of the mitral valve annulus in the ME 4- and 2-chamber and at the apical level to determine the centroid of the LV. Then, mitral annular and endocardial boundary identification was performed by means of the 3D semiautomatic border detection tool. If needed, the end-diastolic and end-systolic contour tracing was manually adjusted to provide the highest quality sequence. After the sequence analysis, the Q-lab software automatically fitted a preconfigured ellipse to the endocardial borders for all the frames in the cardiac loop by computing a mathematically rendered model. The Q-lab software automatically provided parameters of LV global function, that is, end-diastolic volume (EDV), end-systolic volume (ESV), EF, and stroke volume (SV; Fig. 1).

\section{Statistical Analysis}

The a posteriori statistical power was 0.99 for the comparison between 3D and 2D EDV. The following quantities were specified: 0.05 as significance level $(\alpha), 5 \mathrm{~mL}$ as clinically relevant difference $(\delta)$, and $10 \mathrm{~mL}$ as standard deviation of the variable in each group (s).

Continuous variables are presented as mean \pm SD; LV volumes were indexed to the body surface area and expressed as milliliters per square meter. Pairwise differences between 3D and 2D indexed EDV (iEDV) and ESV (iESV), EF, acquisition time (in seconds) and analyses, and differences in repeated $3 \mathrm{D}$ and $2 \mathrm{D} \mathrm{LV}$ indexed volumes were found not to follow a normal or a log-normal distribution (all $P<0.01$ ) when tested with the Shapiro-Wilk method, and thus were analyzed nonparametrically.

The population was categorized according to 2D-derived EF ( $\geq 55 \%$ : normal systolic function; $45 \%$ 54\%: mildly abnormal; 30\%-44\%: moderately abnormal; $<30 \%$ : severely abnormal). ${ }^{17}$ The study population was also divided into patients with normal or dilated LV volume (using an LV iEDV cutoff of $75 \mathrm{~mL} / \mathrm{m}^{2}$ ) based on 2D-derived values. ${ }^{17}$

\section{Statistical Methods Used for Comparison Between LV Volumes and EF Quantified by 3D and 2D TEE}

Means \pm SD and median pairwise differences of $3 \mathrm{D}$ and 2D indexed volumes and EF were calculated in the overall population and in the above-defined subgroups. The $95 \%$ prediction intervals (PIs) of the median pairwise differences were calculated using a binomial method described by Clopper and Pearson ${ }^{19}$ and displayed using a BlandAltman plot. ${ }^{20}$

$2 \mathrm{D}$ volumes were also plotted against $3 \mathrm{D}$ volumes in scatter plots. Scatter plots for 3D versus 2D LV iEDV and iESV were obtained and were analyzed using 3-zone error grid approach, as shown in Figure 2. ${ }^{21}$ The cutoffs defining

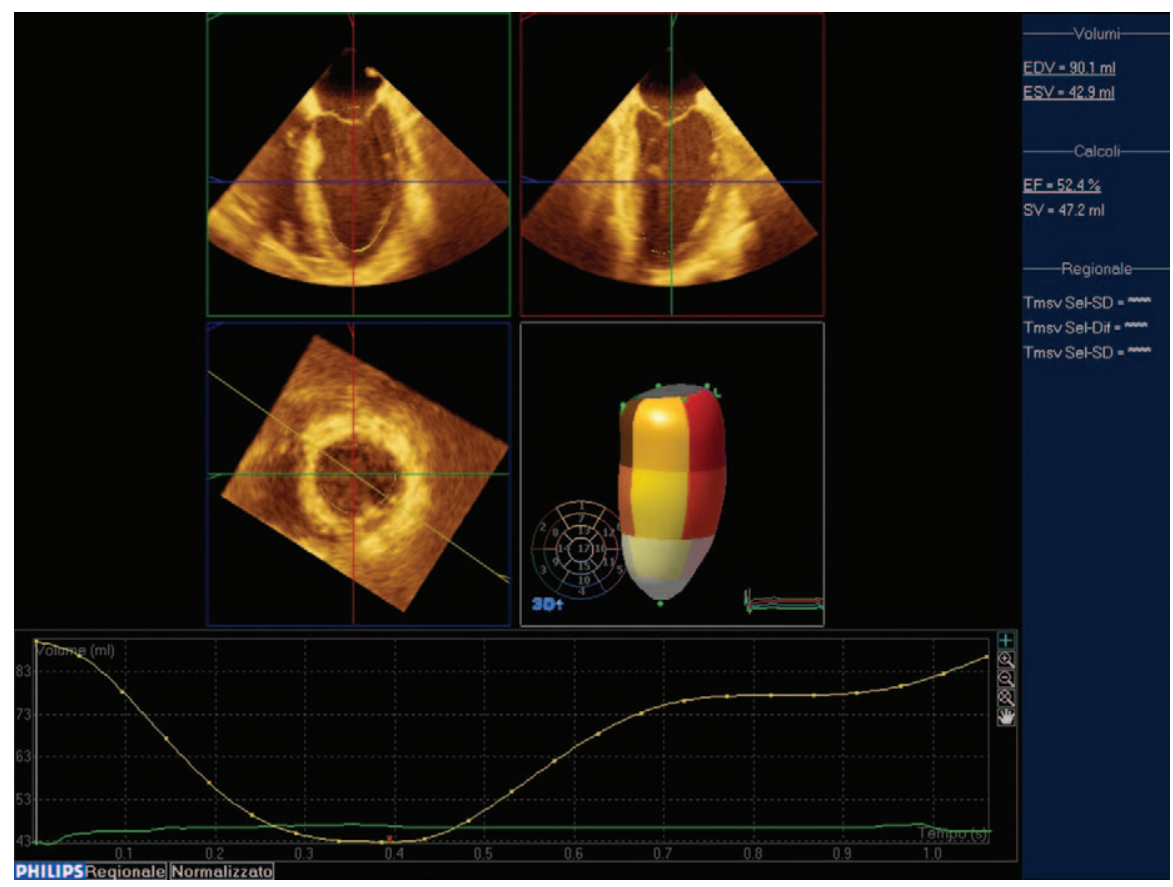

Figure 1. Left ventricular (LV) assessment by Q-lab software. In the graph, on the lower part of the figure, LV volume is plotted versus time, with end-diastolic volume (EDV) as baseline. ESV = endsystolic volume; $E F=$ ejection fraction; $\mathrm{SV}=$ stroke volume. 
A
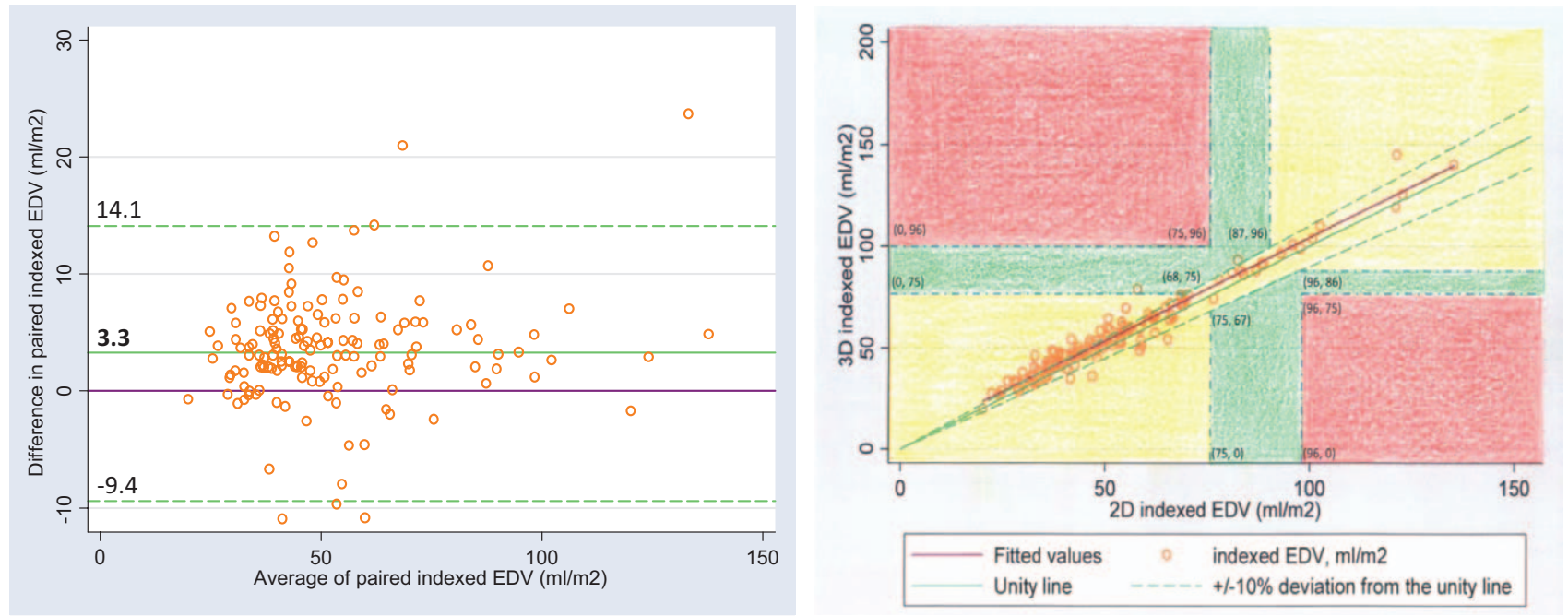

B
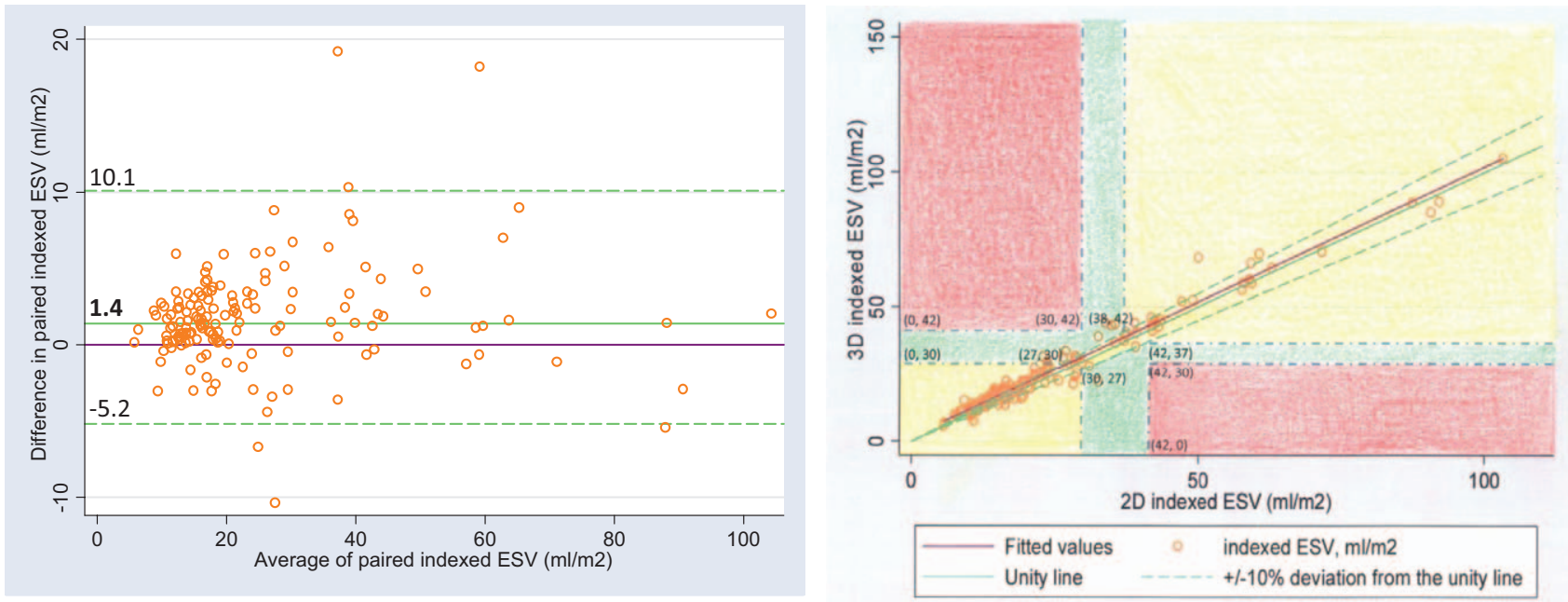

Figure 2. Comparison between 3-dimensional (3D) and 2D left ventricular (LV) indexed end-diastolic volume (EDV; A) and indexed end-systolic volume (ESV; B) in the study population $(n=152)$. These values were highly correlated, but 3D volumes were slightly larger than $2 \mathrm{D}$ volumes. On the left, Bland-Altman plot of the difference in 152 paired LV indexed volumes on the ordinate against the average of the LV indexed volumes on the abscissa. The observed median agreement is displayed as continuous green line, and the $95 \%$ prediction intervals are displayed as dashed green lines. On the right, scatter plots of paired LV indexed volumes with 3-zone error grid. ${ }^{21}$ Indexed ventricular volumes obtained with the 2D (abscissa) and 3D transesophageal echocardiography (ordinate) were plotted as ordered pairs. The coordinates of the corners of the boxes are showed between brackets. Refer to text in the Methods section for more details.

the 3 zones (yellow, green, orange) were chosen according to the current guidelines: $\mathrm{iEDV}>96 \mathrm{~mL} / \mathrm{m}^{2}$ and/or $\mathrm{iESV}$ $>42 \mathrm{~mL} / \mathrm{m}^{2}$ were defined as severely dilated, while iEDV $\leq 75 \mathrm{~mL} / \mathrm{m}^{2}$ and / or iESV $\leq 30 \mathrm{~mL} / \mathrm{m}^{2}$ were considered normal. ${ }^{17}$ The purple line represents our fitted values of $3 \mathrm{D}$ volumes versus 2D volumes; the green line represents the perfect correlation between $3 \mathrm{D}$ and $2 \mathrm{D}$ measurements. The dashed green lines represent a deviation of $\pm 10 \%$ of $3 \mathrm{D}$ $\mathrm{LV}$ indexed volumes from the $2 \mathrm{D}$ reference. Yellow zone was defined by a lowermost section for iEDV $\leq 75 \mathrm{~mL} / \mathrm{m}^{2}$ or iESV $\leq 30 \mathrm{~mL} / \mathrm{m}^{2}$, an isthmus delimited by the abovedefined dashed green lines, and an uppermost region for iEDV $>96 \mathrm{~mL} / \mathrm{m}^{2}$ or $\mathrm{iESV}>42 \mathrm{~mL} / \mathrm{m}^{2}$. The orange zone has an uppermost section for $2 \mathrm{D} \mathrm{iEDV} \leq 75 \mathrm{~mL} / \mathrm{m}^{2}$ but $3 \mathrm{D}$ $\mathrm{iEDV}>96 \mathrm{~mL} / \mathrm{m}^{2}$ or $2 \mathrm{D} \mathrm{iESV} \leq 30 \mathrm{~mL} / \mathrm{m}^{2}$ but $3 \mathrm{D} \mathrm{iESV}>42$ $\mathrm{mL} / \mathrm{m}^{2}$ and a lowermost region for $2 \mathrm{D} \mathrm{iEDV}>96 \mathrm{~mL} / \mathrm{m}^{2}$ but $3 \mathrm{D} \mathrm{iEDV} \leq 75 \mathrm{~mL} / \mathrm{m}^{2}$ or $2 \mathrm{D} \mathrm{iESV}>42 \mathrm{~mL} / \mathrm{m}^{2}$ but $3 \mathrm{D}$ $\mathrm{iESV} \leq 30 \mathrm{~mL} / \mathrm{m}^{2}$. Green zone is the area between yellow and orange zones.

The equality of matched pairs of $3 \mathrm{D}$ and $2 \mathrm{D}$ indexed volumes and EF in the global population and in the abovedefined subgroups was tested using the Wilcoxon matchedpairs signed-ranks test (with Bonferroni correction where appropriate). The 99\% confidence intervals (CIs) median pairwise differences between 3D and 2D indexed volumes and EF were calculated as well, even though these CI may be misleading because their distributions were neither normal nor log normal.

A nonparametric test for trend across ordered groups developed by $\mathrm{Cuzic}^{22}$ was used to test 3D-derived SV across the above-defined subgroups of EF. 
Statistical Methods Used for Comparing the 3D and 2D Image Quality and Time Requirement for Acquisition and Analysis

Absolute number and percentage of patients were displayed in the 4-point scale of image quality, as defined in Table 2. Means \pm SD of 3D and 2D acquisition time and analysis time were calculated and displayed in box plot graphs, together with median and 25 th and 75 th percentiles.

The Wilcoxon matched-pairs signed-ranks test was used to compare 3D and 2D image quality, as well as the $3 \mathrm{D}$ versus $2 \mathrm{D}$ time requirements. The pairwise differences between 3D and 2D acquisition and analysis time and their corresponding 95\% PIs were calculated. ${ }^{19}$ Manual correction of the 3D borders between patients with normal ( $\geq 55 \%)$ and reduced $(<55 \%)$ EF was expressed as percentage and compared using the Pearson $\chi^{2}$ test.

\section{Statistical Methods Used for Comparing the 3D and 2D Intra- and Interobserver Reproducibility for Quantification of LV Volumes}

Intraobserver reproducibility for $3 \mathrm{D}$ and $2 \mathrm{D} \mathrm{LV}$ indexed volumes was tested on a subgroup of 30 patients randomly selected. To assess interobserver reproducibility, measurements of the 3D and 2D LV volumes were repeated in all patients by a second operator certified for TEE examinations who was blinded to the measurements of the first operator. Differences in repeated LV indexed volume measurements were plotted against their average using a Bland-Altman plot to graphically represent the inter- and intrareproducibility for iEDV and iESV. ${ }^{20,23}$ The corresponding lines based on locally weighted polynomial regression were displayed both for 3D and 2D values in the same graph. Differences in repeated 3D LV indexed volumes were compared against differences in repeated 2D LV indexed volumes using the Wilcoxon matched-pairs signed-ranks test. The pairwise differences of repeated 3D indexed volumes versus repeated 2D indexed volumes and their corresponding 95\% PIs were calculated. ${ }^{19}$

$3 \mathrm{D}$ and $2 \mathrm{D}$ reproducibility were also expressed with the coefficient of variation, defined as the ratio of the SD to the

\begin{tabular}{lc} 
Table 3. Demographics and Type of Surgery \\
General population $(\boldsymbol{n}=\mathbf{1 5 2})$ \\
Age, $y$ & $68.2 \pm 10.7$ \\
Female, $n(\%)$ & $38(25)$ \\
Weight, $\mathrm{kg}$ & $77.1 \pm 14.2$ \\
Height, $\mathrm{cm}$ & $168.6 \pm 8.2$ \\
$\mathrm{BMI}, \mathrm{kg} / \mathrm{m}^{2}$ & $27.1 \pm 4.5$ \\
$\mathrm{BSA}, \mathrm{m}^{2}$ & $1.89 \pm 0.20$ \\
Presurgery EF, \% & $57 \pm 12$ \\
Type of cardiac surgery, $n(\%)$ & $174^{\mathrm{a}}$ \\
CABG & $115(76)$ \\
Valvular surgery & \\
$\quad$ Mitral valve & $18(12)$ \\
Aortic valve & $32(21)$ \\
Aortic root surgery & $9(6)$ \\
\hline
\end{tabular}

$\mathrm{BMI}=$ body mass index; $\mathrm{BSA}=$ body surface area; $\mathrm{EF}=$ ejection fraction; $\mathrm{CABG}=$ coronary artery bypass surgery.

aThe majority of the patients underwent CABG or isolated valvular surgery or isolated aortic root surgery. Twenty-two patients (14\%) underwent a combined cardiac surgery (CABG and valvular surgery or aortic valve and aortic root surgery), which explains why the total number of surgeries amounts to 152 $+22=174$. mean of the difference between 2 repeated measurements per subject. Coefficients of variation for 3D and 2D measurements were compared using the Wilcoxon matchedpairs signed-ranks test.

Data analyses were performed using STATA software (StataCorp, College Station TX) and Excel (Microsoft, Redmond, WA). A $P$ value $<0.05$ was considered statistically significant.

\section{RESULTS}

\section{Volumes and Function}

The clinical characteristics of the study population are shown in Table 3. Bland-Altman plots of 3D versus 2D iEDV (Fig. 2A) and iESV (Fig. 2B) are shown in Figure 2, with median pairwise differences and 95\% PIs. The observed median agreement is above the 0 line, which represents perfect matching; since the PIs include the 0 value, by repeating the same LV measurements many times in individual patients, it would be expected to obtain on average $3 \mathrm{D}$ volumes larger than 2D volumes, but the opposite situation may also occur. The same results in terms of median, pairwise differences and 95\% PIs were also obtained in the predefined subgroups of patients (i.e., normal, mildly abnormal, moderately abnormal, or severely abnormal EF and normal or dilated ventricular dimensions), as shown in Figure 3.

On the right side of Figure 2, the scatter plots of paired LV indexed volumes are displayed together with 3-zone error grid analyses. ${ }^{21}$ The line of the fitted indexed volumes (purple) is above the line of the perfect correlation between 3D and 2D (green). Within the yellow zone of the 3-zone error grid, the discrepancies between 2D and 3D measurements have fewer clinical consequences than in the other zones. In the large uppermost and lowermost regions of yellow zone, even large discrepancies between 3D and 2D may occur without affecting the LV classification as severely dilated or normal, respectively. On the contrary, in the isthmus yellow section, 3D and 2D measurements must most closely agree. Therefore, the isthmus is the critical area, where even small discrepancies between 2D and 3D measurements may lead to a misclassification of the severity of the LV enlargement with possible clinical consequences. Most of the dots in Figure 2 (150 of 152 dots for iEDV and 141 of 152 dots for iESV) are plotted within this yellow zone. Dots in the orange zone would represent severe discrepancies between 2D and 3D measurements leading to a relevant misclassification of the LV dilation (e.g., LV classified as normal by 3D and as severely dilated by 2D); no dots in Figure 2 are plotted within the orange zone. The green zone represents some discrepancy in LV volumes between 2D and 3D (not as severe as in the orange zone). A minority of the dots is plotted in this zone ( 2 of 152 dots for $i E D V, 1.3 \%$, and 11 of 152 dots for $\mathrm{iESV}, 7.2 \%$ ).

As shown in Table 4, there was no significant difference in EF measured by 3D and 2D TEE (median pairwise difference, $-0.4 \%$ [95\% PIs, $-8.6 \%$ to $8.8 \%$ ]; $P=0.227$ ). Conversely, the LV indexed volumes measured by 3D echocardiography were significantly higher than those measured by the $2 \mathrm{D}$ echocardiography in the entire population. 
A
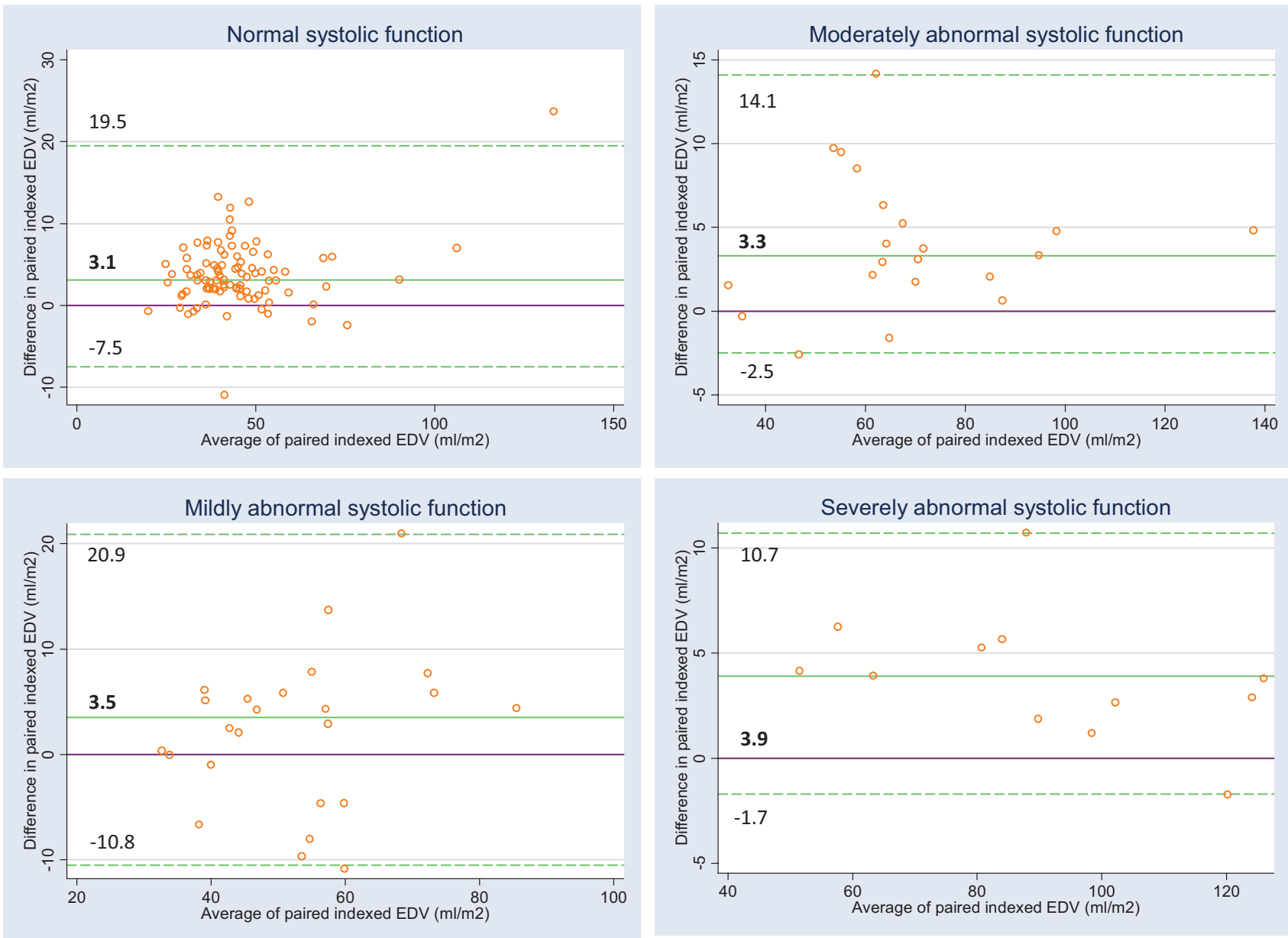

B
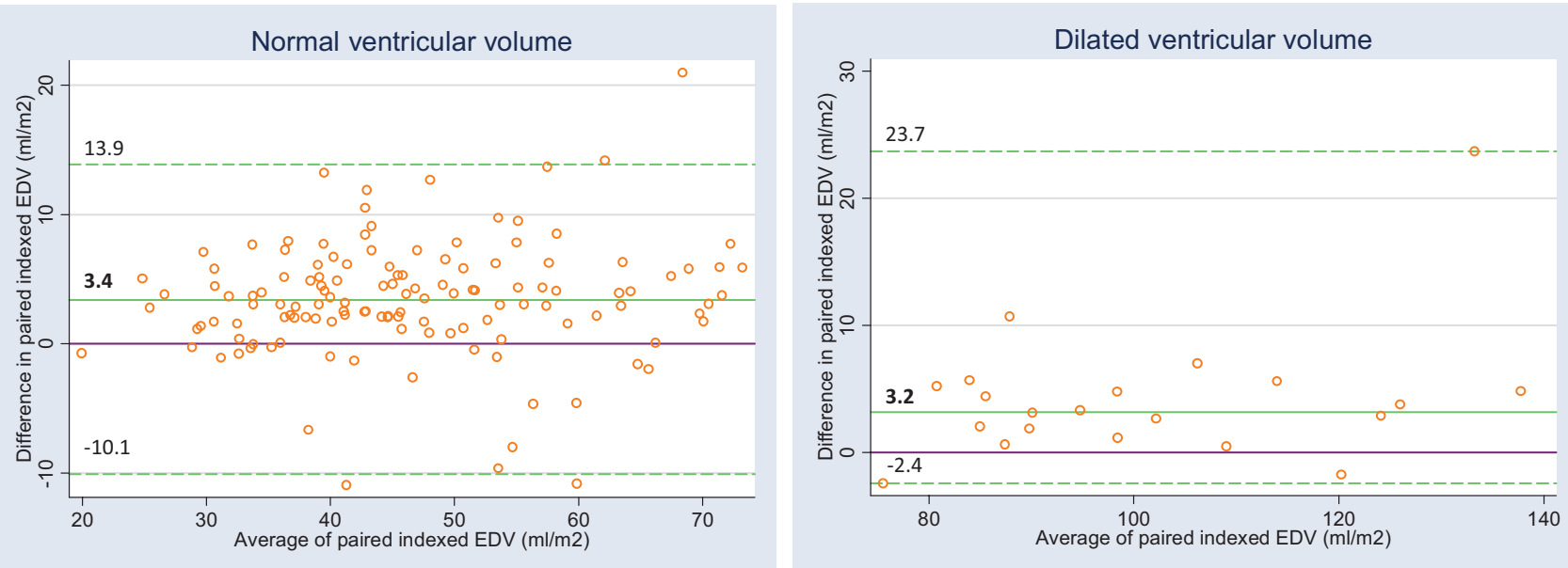

Figure 3. Bland-Altman plot of the 3-dimensional (3D) left ventricular (LV) indexed end-diastolic volume (EDV) against the 2D LV indexed endsystolic volume (ESV) in the stratification done by subgroups: normal, mildly abnormal, moderately abnormal, or severely abnormal ejection fraction (A) and normal or dilated ventricular dimensions (B). The observed median agreement is displayed as continuous green line, and the 95\% prediction intervals are displayed as dashed green lines.

The same results were also confirmed in the stratification done by subgroups: normal, mildly abnormal, moderately abnormal, or severely abnormal EF and normal or dilated ventricular dimensions.
Three-dimensional-derived SV in the global population was $54 \pm 20 \mathrm{~mL}$ and was progressively larger across the EF subgroups according to increase in EF $(44 \pm 12 \mathrm{~mL}$ in the severely abnormal systolic function subgroup; $51 \pm 16 \mathrm{~mL}$ 


\begin{tabular}{|c|c|c|c|c|}
\hline & 3D TEE & 2D TEE & $P$ value & Median pairwise difference (99\% Cl) \\
\hline$E F, \%$ & $55 \pm 14$ & $55 \pm 14$ & 0.227 & $-0.4(-1.2$ to 0.3$)$ \\
\hline $\mathrm{iEDV}, \mathrm{mL} / \mathrm{m}^{2}$ & $54 \pm 21$ & $51 \pm 21$ & $<0.001$ & 3.3 (2.5 to 4.2$)$ \\
\hline $\mathrm{iESV}, \mathrm{mL} / \mathrm{m}^{2}$ & $26 \pm 18$ & $24 \pm 17$ & $<0.001$ & $1.4(1.0$ to 2.0$)$ \\
\hline \multicolumn{5}{|c|}{$\begin{array}{l}3 \mathrm{D} \text { and } 2 \mathrm{D} \mathrm{iEDV} \text { in } \mathrm{mL} / \mathrm{m}^{2} \text { in subgroups of patients (the percentage of the entire population represented in each subgroup is reported in brackets), } \\
\text { according to the following: }\end{array}$} \\
\hline \multicolumn{5}{|c|}{ Systolic function } \\
\hline Normal (62\%) & $47 \pm 16$ & $43 \pm 15$ & $<0.001$ & $3.2(2.3$ to 4.4$)$ \\
\hline Mildly abnormal (16\%) & $53 \pm 14$ & $51 \pm 12$ & 0.794 & $3.6(-3.8$ to 5.8$)$ \\
\hline Moderately abnormal (14\%) & $71 \pm 23$ & $67 \pm 23$ & 0.001 & 3.3 (1.6 to 6.2$)$ \\
\hline Severely abnormal (8\%) & $89 \pm 23$ & $85 \pm 24$ & 0.023 & $3.9(0.7$ to 6.9$)$ \\
\hline \multicolumn{5}{|l|}{ Ventricular volume } \\
\hline Normal (86\%) & $48 \pm 12$ & $45 \pm 12$ & $<0.001$ & 3.5 (2.4 to 4.2$)$ \\
\hline Dilated (14\%) & $101 \pm 19$ & $96 \pm 17$ & 0.002 & $3.2(1.2$ to 5.6$)$ \\
\hline
\end{tabular}

$\mathrm{Cl}=$ confidence interval; $\mathrm{EF}=$ ejection fraction; $\mathrm{iEDV}=$ indexed end-diastolic volume; iESV = indexed end-systolic volume; TEE = transesophageal echocardiography.
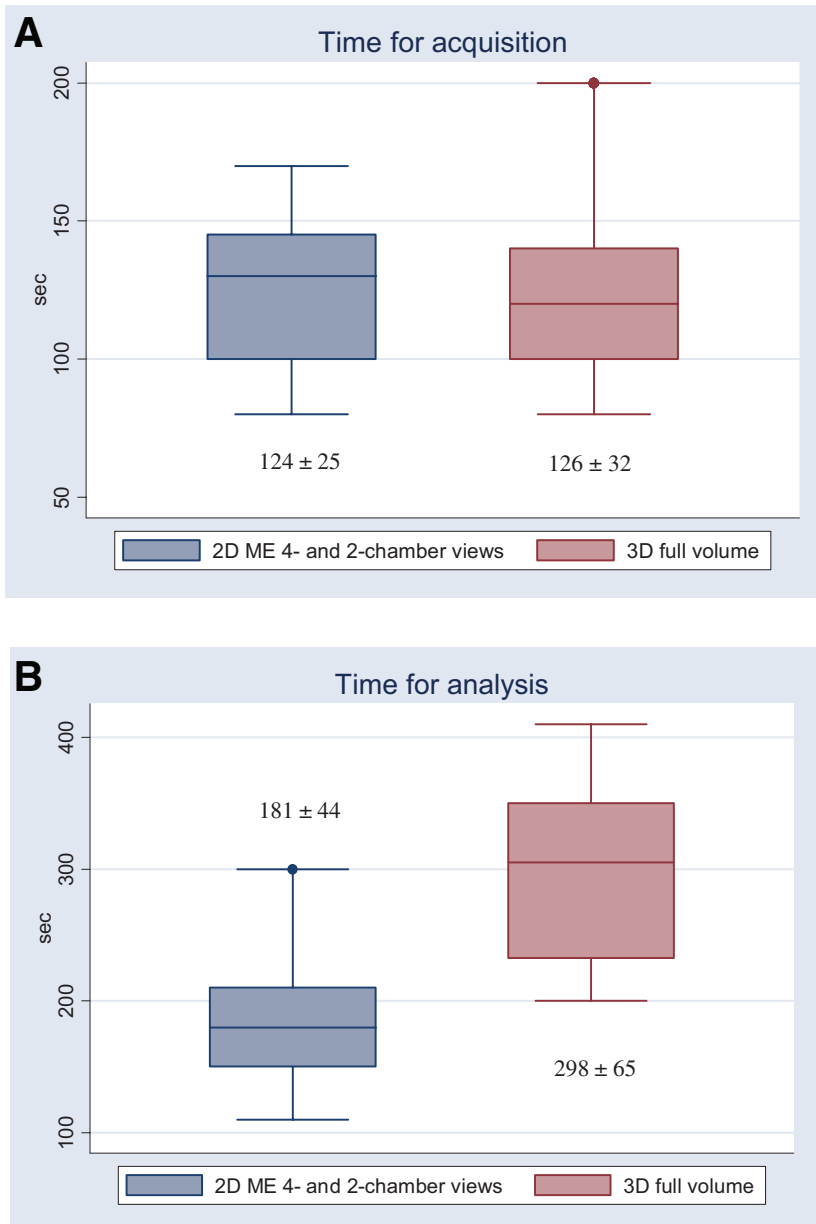

Figure 4. The box plot graphs show the acquisition time $(\mathrm{A})$ and the analysis time (B) of 2-dimensional (2D) midesophageal (ME) 4- and 2-chamber views (blue boxes) and 3D left ventricular full volume (red boxes). The boxes are determined by the 25th $=\mathrm{Q} 1$ and 75 th $=\mathrm{Q} 3$ percentiles, and the line in the middle of the box is the median; the lower whisker indicates Q1 - 1.5 $\times(\mathrm{Q} 3-\mathrm{Q} 1)$, and the upper whisker indicates Q3 $+1.5 \times(\mathrm{Q} 3-\mathrm{Q} 1)$. The numbers showed represent the mean \pm SD.

in moderately abnormal systolic function; $52 \pm 18 \mathrm{~mL}$ in mildly abnormal systolic function; $57 \pm 21 \mathrm{~mL}$ in the normal systolic function subgroups; $P=0.024)$.

\section{Image Quality and Time Requirement}

As shown in Table 2, the quality of 3D TEE images was optimal in more than half of the study population and resulted in similar $2 \mathrm{D}$ image quality $(P=0.206)$. It was necessary to repeat the $2 \mathrm{D}$ acquisition in 23 patients (for a total of $322 \mathrm{D}$ images) and the 3D acquisition in 19 patients to improve the image quality.

The time required for 2D and 3D imaging is compared in Figure 4. There was no difference in the time required for acquisition of $3 \mathrm{D}$ and $2 \mathrm{D}$ images $(P=0.805$; pairwise difference $=2$ seconds $[95 \%$ PIs, -20 to 35 seconds]), but analysis of $3 \mathrm{D}$ images required significantly more time than $2 \mathrm{D}$ images $(P<0.001$; pairwise difference $=117$ seconds $[95 \%$ PIs, 66 to 197 seconds]). A manual correction of the 3D automated border detection was required in 59\% of the 3D TEE images in $73 \%$ of patients with abnormal EF and in $52 \%$ of those with normal EF $(P=0.009)$, especially at the level of the lateral apex (in 57 patients, 37\%) and inferior apex (in 45 patients, 29\%).

\section{Reproducibility}

Figure 5 presents the inter- and intrareproducibility analyses for $\mathrm{iEDV}$ and $\mathrm{iESV}$. In each graph, the distribution of the 3D and 2D measurements and the corresponding lines based on locally weighted polynomial regression are within the same range of values. Differences in repeated $3 \mathrm{D}$ versus $2 \mathrm{D}$ indexed volumes were not statistically significant, both considering interobserver reproducibility (iEDV: $P=0.125$; pairwise difference, $0.26 \pm 1.76 \mathrm{~mL}[95 \%$ PIs, -3.58 to $3.73 \mathrm{~mL}$ ] and iESV: $P=0.126$; pairwise difference, $-0.16 \pm 1.67 \mathrm{~mL}$ [95\% PIs, -3.96 to $3.69 \mathrm{~mL}$ ) and intraobserver reproducibility (iEDV: $P=0.975$; pairwise difference, $-0.02 \pm 1.20 \mathrm{~mL}$ [95\% PIs, -2.32 to $2.08 \mathrm{~mL}$ ] and iESV: $P=0.228$; pairwise difference, $-0.19 \pm 1.13 \mathrm{~mL}[95 \%$ PIs, -2.47 to $2.53 \mathrm{~mL}]$ ).

Considering interobserver reproducibility, we found coefficients of variation of $3.2 \%$ for $3 \mathrm{D}$ iEDV versus $2.9 \%$ for $2 \mathrm{D}$ iEDV $(P=0.195)$ and $7.9 \%$ for $3 \mathrm{D}$ iESV versus $8.9 \%$ for $2 \mathrm{D}$ iESV $(P=0.165)$. Considering intraobserver reproducibility, the calculated coefficients of variation were $2.0 \%$ for 3D iEDV compared with $2.2 \%$ for $2 \mathrm{D}$ iEDV $(P=0.781)$ and $4.1 \%$ for $3 \mathrm{D}$ iESV compared with $4.7 \%$ for $2 \mathrm{D}$ iESV $(P=0.280)$. 

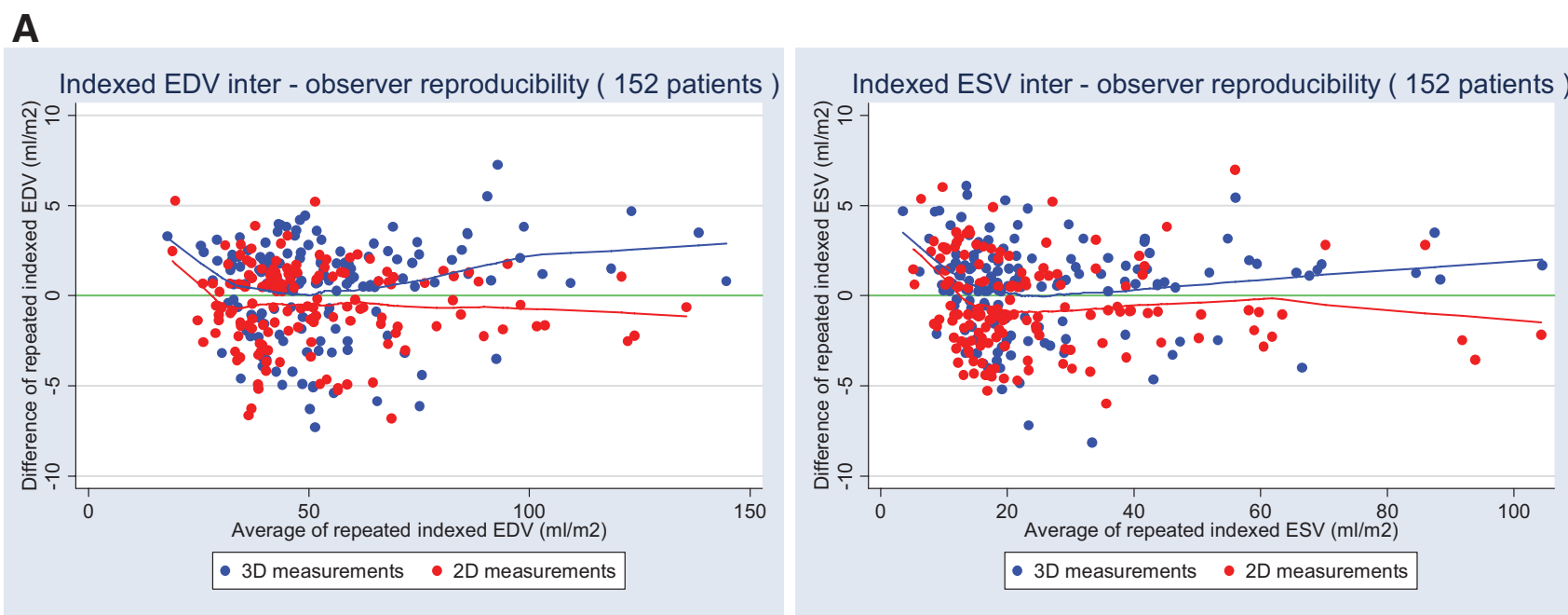

B
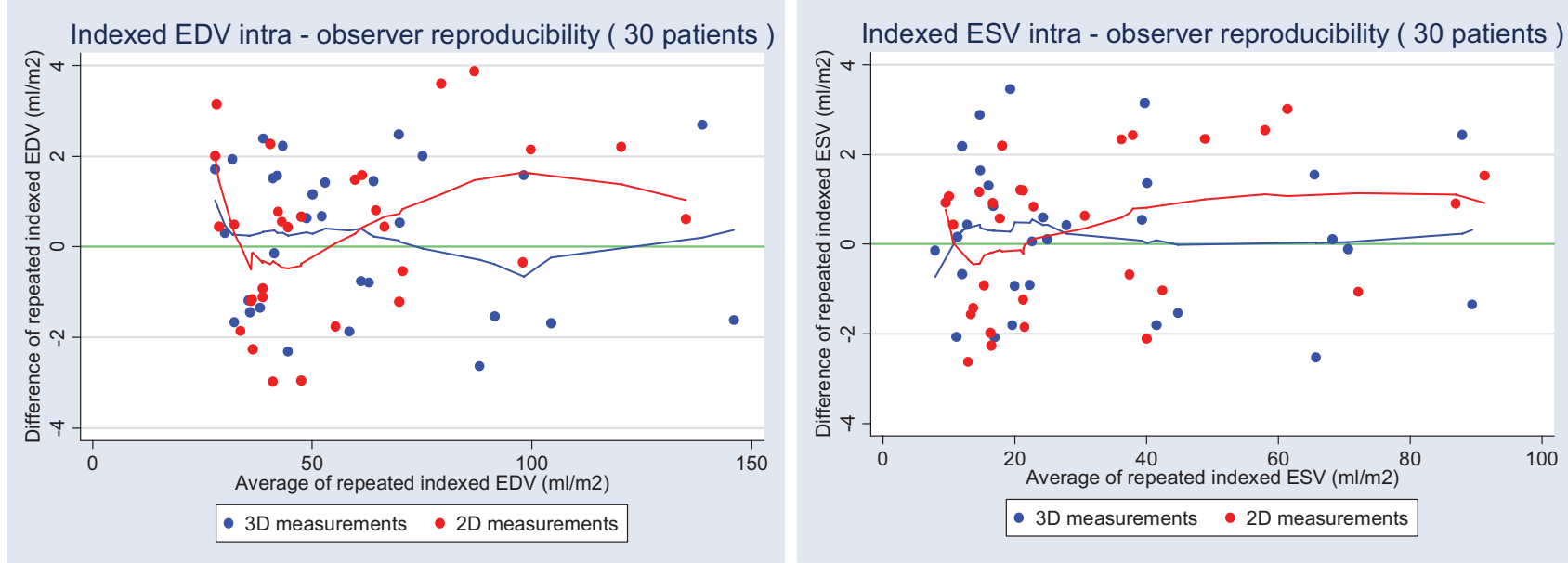

Figure 5. Bland-Altman plot for interobserver (A) and intraobserver reproducibility (B) of indexed end-diastolic volume (EDV; left side) and indexed end-systolic volume (ESV; right side). Dots in blue represent 3-dimensional (3D) measurements, and dots in red represent 2D measurements. The corresponding lines are based on locally weighted polynomial regression.

\section{DISCUSSION}

In the present study, we compared intraoperative 3D with 2D TEE for the evaluation of LV volumes and EF. Previous TTE studies have focused on 3D LV assessment, suggesting that $3 \mathrm{D}$ is more precise and accurate for LV volume quantification than $2 \mathrm{D}$ and offers reproducible information. ${ }^{4,9}$ However, less information is available regarding 3D TEE in the perioperative setting. In this study, we noted larger 3D LV volumes than 2D LV volumes and that 3D TEE needed longer analysis time with respect to $2 \mathrm{D}$. Our results showed no difference in terms of LV EF, image quality, and reproducibility between 3D and 2D TEE.

\section{Volumes and Function}

Our results showed a difference between LV indexed volumes measured by 3D and 2D TEE as 3D volumes were larger than 2D volumes. This difference appeared statistically significant when tested by the Wilcoxon matchedpairs signed-ranks test and expressed as CIs of median pairwise differences. However, the median pairwise difference between $3 \mathrm{D}$ versus $2 \mathrm{D}$ volumes is on the order of a few milliliters (i.e., $3.3 \mathrm{~mL} / \mathrm{m}^{2}$ for $\mathrm{iEDV}$ and $1.4 \mathrm{~mL} / \mathrm{m}^{2}$ for iESV) and the PIs included the 0 value, indicating that the clinical relevance of these differences is small. This consideration is also supported by 3-zone error grid analysis of the LV indexed volumes showing that the difference between 3D and 2D measurements does not affect the LV classification as normal, mildly to moderately dilated, or severely dilated.

With respect to $\mathrm{LV}$ function, there were no differences between 3D and 2D EF, suggesting that the evaluation of LV global function by 3D TEE is comparable with 2D TEE. As EF is calculated from a simple algebraic equation ([EDV - ESV]/EDV), a systematic upshift of 3D LV volumes versus 2D LV volumes leads to a cancelation in the differences between $3 \mathrm{D}$ and $2 \mathrm{D}$ EF.

The 3D echocardiography also allowed us to assess the LV function by means of 3D-derived SV. Quantification of LV cardiac output by 3D TTE echocardiography has been validated against thermodilution ${ }^{24}$ or MRI. ${ }^{11}$ A good correlation between 3D-derived SV and thermodilution was reported by Culp et al. ${ }^{25}$ However, there were significant bias and wide limits of agreement, limiting the overall accuracy of the 3D TEE measurements. In our study, we found 
that 3D-derived SV progressively decreased according to worsening in 2D-derived EF.

\section{Image Quality and Time Requirements}

The success rate for 3D image acquisition and analysis was equal to that of 2D echocardiography. The visualization of LV endocardium by 3D TEE was optimal to good in $85 \%$ of our study population, in line with encouraging data reported in the literature. ${ }^{26}$ In this study, 3D image quality was similar to $2 \mathrm{D}$, the latter also being similar to previous data in the literature on endocardial visualization with 2D TEE. ${ }^{27}$

Although acquisition times were similar in 3D and 2D, $3 \mathrm{D}$ analysis to obtain $\mathrm{LV}$ volumes and $\mathrm{EF}$ required more time than 2D, and this could be a disadvantage of the use of $3 \mathrm{D}$ in the operating room. However, the 5-minute average needed to analyze $\mathrm{LV}$ with $3 \mathrm{D}$ was still compatible with the pace of our operating room activity. Moreover, 3D provides a complete characterization of LV including information on regional function, ${ }^{15}, 28$ which would need additional time with 2D TEE. Although not the topic of the present study, evaluation of LV regional function is of potential importance during cardiac surgery to facilitate detection of myocardial ischemia.

\section{Reproducibility}

Our data on intra- and interobserver reproducibility for 3D indexed volumes were satisfactory, and 3D reproducibility was similar to $2 \mathrm{D}$ reproducibility ${ }^{29}$ but failed to be clearly superior in contrast to TTE studies. . $^{4,10,11}$ These discordant results may be partly explained by the fact that our measurements were performed on TEE, unlike the previous TTE studies.

\section{Limitations}

Some limitations of this study should be noted. First, 3D LV measurements in our patients were compared only with 2D values, both acquired with TEE and, as is well known, transesophageal imaging techniques involve difficulties in the visualization of the apex and lateral heart wall. A comparison with reference techniques such as MRI or 3D TTE would be necessary for final validation of 3D TEE measures. For this purpose, studies of 3D TEE in the echo laboratory appear to be more appropriate, being performed in hemodynamic conditions similar to the reference technique. Intraoperatively, we could not perform 3D TTE in the same hemodynamic conditions as TEE for technical reasons (surgical preparation was underway and a cardiologist trained in 3D TTE acquisition was not available). The validation of 3D TEE volumes measured intraoperatively against pre- or postoperative MRI would be logistically challenging. Second, no comparison between LV assessed by Philips Q-lab 3D-Advanced software and different 3D software was done, and 3D-derived SV was not compared with thermodilution. Third, this study was performed in patients undergoing elective cardiac surgery having hemodynamically stable conditions and sinus rhythm. Finally, a single operator acquired and analyzed both 2D and 3D images. Therefore, bias cannot be excluded in performing 2D and 3D measurements and analyses. However, all measurements were repeated by a second experienced operator and interobserver differences were small and not statistically significant.

In conclusion, intraoperative 3D TEE of LV demonstrated no differences compared with 2D TEE in terms of LV EF, image quality, and reproducibility. The 3D required more time compared with $2 \mathrm{D}$ TEE, and ventricular volumes measured by 3D TEE were larger than those obtained from 2D TEE. However, the difference between $3 \mathrm{D}$ and $2 \mathrm{D}$ volumes was on the order of a few milliliters and did not affect the classification of LV as normal, mildly to moderately dilated, or severely dilated using the 3-zone grid analysis, thus limiting the clinical relevance of these differences.

\section{DISCLOSURES}

Name: Alessandra Meris, MD.

Contribution: This author helped design the study, conduct the study, analyze the data, and write the manuscript.

Attestation: Alessandra Meris has seen the original study data, reviewed the analysis of the data, approved the final manuscript, and is the author responsible for archiving the study files.

Name: Luisa Santambrogio, MD

Contribution: This author helped design the study, conduct the study, and write the manuscript.

Attestation: Luisa Santambrogio has seen the original study data, reviewed the analysis of the data, and approved the final manuscript.

Name: Gabriele Casso, MD.

Contribution: This author helped design the study and conduct the study.

Attestation: Gabriele Casso approved the final manuscript.

Name: Romano Mauri, MD

Contribution: This author helped conduct the study.

Attestation: Romano Mauri approved the final manuscript.

Name: Albin Engeler, MD.

Contribution: This author helped conduct the study.

Attestation: Albin Engeler approved the final manuscript.

Name: Tiziano Cassina, MD.

Contribution: This author helped design the study, conduct the study, and write the manuscript.

Attestation: Tiziano Cassina has seen the original study data, reviewed the analysis of the data, and approved the final manuscript.

This manuscript was handled by: Martin J. London, MD.

\section{ACKNOWLEDGMENTS}

We are very grateful for the statistical support provided by the Institut für Datenanalyse und Prozessdesign Zürcher Hochschule für angewandte Wissenschaften and to our nurses for their fruitful teamwork not only for this study, but also every day in the operating room.

\section{REFERENCES}

1. Savage RM, Lytle BW, Aronson S, Navia JL, Licina M, Stewart WJ, Starr NJ, Loop FD. Intraoperative echocardiography is indicated in high-risk coronary artery bypass grafting. Ann Thorac Surg 1997;64:368-73; discussion 373-4

2. Desjardins G, Cahalan M. The impact of routine trans-oesophageal echocardiography (TOE) in cardiac surgery. Best Pract Res Clin Anaesthesiol 2009;23:263-71 
3. Shanewise JS, Cheung AT, Aronson S, Stewart WJ, Weiss RL, Mark JB, Savage RM, Sears-Rogan P, Mathew JP, Quiñones MA, Cahalan MK, Savino JS. ASE/SCA guidelines for performing a comprehensive intraoperative multiplane transesophageal echocardiography examination: recommendations of the American Society of Echocardiography Council for Intraoperative Echocardiography and the Society of Cardiovascular Anesthesiologists Task Force for Certification in Perioperative Transesophageal Echocardiography. Anesth Analg 1999;89:870-84

4. Jacobs LD, Salgo IS, Goonewardena S, Weinert L, Coon P, Bardo D, Gerard O, Allain P, Zamorano JL, de Isla LP, Mor-Avi V, Lang RM. Rapid online quantification of left ventricular volume from real-time three-dimensional echocardiographic data. Eur Heart J 2006;27:460-8

5. Mor-Avi V, Lang RM. The use of real-time three-dimensional echocardiography for the quantification of left ventricular volumes and function. Curr Opin Cardiol 2009;24:402-9

6. Gutiérrez-Chico JL, Zamorano JL, Pérez de Isla L, Orejas M, Almería C, Rodrigo JL, Ferreirós J, Serra V, Macaya C. Comparison of left ventricular volumes and ejection fractions measured by three-dimensional echocardiography versus by two-dimensional echocardiography and cardiac magnetic resonance in patients with various cardiomyopathies. Am J Cardiol 2005;95:809-13

7. Kühl HP, Schreckenberg M, Rulands D, Katoh M, Schäfer W, Schummers G, Bücker A, Hanrath P, Franke A. High-resolution transthoracic real-time three-dimensional echocardiography: quantitation of cardiac volumes and function using semi-automatic border detection and comparison with cardiac magnetic resonance imaging. J Am Coll Cardiol 2004;43:2083-90

8. Mor-Avi V, Sugeng L, Weinert L, MacEneaney P, Caiani EG, Koch R, Salgo IS, Lang RM. Fast measurement of left ventricular mass with real-time three-dimensional echocardiography: comparison with magnetic resonance imaging. Circulation 2004;110:1814-8

9. Buck T, Hunold P, Wentz KU, Tkalec W, Nesser HJ, Erbel R. Tomographic three-dimensional echocardiographic determination of chamber size and systolic function in patients with left ventricular aneurysm: comparison to magnetic resonance imaging, cineventriculography, and two-dimensional echocardiography. Circulation 1997;96:4286-97

10. Chuang ML, Hibberd MG, Salton CJ, Beaudin RA, Riley MF, Parker RA, Douglas PS, Manning WJ. Importance of imaging method over imaging modality in noninvasive determination of left ventricular volumes and ejection fraction: assessment by two- and three-dimensional echocardiography and magnetic resonance imaging. J Am Coll Cardiol 2000;35:477-84

11. Mor-Avi V, Jenkins C, Kühl HP, Nesser HJ, Marwick T, Franke A, Ebner C, Freed BH, Steringer-Mascherbauer R, Pollard H, Weinert L, Niel J, Sugeng L, Lang RM. Real-time 3-dimensional echocardiographic quantification of left ventricular volumes: multicenter study for validation with magnetic resonance imaging and investigation of sources of error. JACC Cardiovasc Imaging 2008;1:413-23

12. Vegas A, Meineri M. Core review: three-dimensional transesophageal echocardiography is a major advance for intraoperative clinical management of patients undergoing cardiac surgery: a core review. Anesth Analg 2010;110:1548-73

13. Mackensen GB, Swaminathan M, Mathew JP. PRO editorial: PRO: three-dimensional transesophageal echocardiography is a major advance for intraoperative clinical management of patients undergoing cardiac surgery. Anesth Analg 2010;110:1574-8

14. D'Ambra MN. CON editorial: CON: three-dimensional transesophageal echocardiography is a major advance for intraoperative clinical management of patients undergoing cardiac surgery. Anesth Analg 2010;110:1579-80

15. Sugeng L, Shernan SK, Salgo IS, Weinert L, Shook D, Raman J, Jeevanandam V, Dupont F, Settlemier S, Savord B, Fox J,
Mor-Avi V, Lang RM. Live 3-dimensional transesophageal echocardiography initial experience using the fully-sampled matrix array probe. J Am Coll Cardiol 2008;52:446-9

16. Cerqueira MD, Weissman NJ, Dilsizian V, Jacobs AK, Kaul S, Laskey WK, Pennell DJ, Rumberger JA, Ryan T, Verani MS; American Heart Association Writing Group on Myocardial Segmentation and Registration for Cardiac Imaging. Standardized myocardial segmentation and nomenclature for tomographic imaging of the heart. A statement for healthcare professionals from the Cardiac Imaging Committee of the Council on Clinical Cardiology of the American Heart Association. Circulation 2002;105:539-42

17. Lang RM, Bierig M, Devereux RB, Flachskampf FA, Foster E, Pellikka PA, Picard MH, Roman MJ, Seward J, Shanewise JS, Solomon SD, Spencer KT, Sutton MS, Stewart WJ; Chamber Quantification Writing Group; American Society of Echocardiography's Guidelines and Standards Committee; European Association of Echocardiography. Recommendations for chamber quantification: a report from the American Society of Echocardiography's Guidelines and Standards Committee and the Chamber Quantification Writing Group, developed in conjunction with the European Association of Echocardiography, a branch of the European Society of Cardiology. J Am Soc Echocardiogr 2005;18:1440-63

18. Corsi C, Lang RM, Veronesi F, Weinert L, Caiani EG, MacEneaney P, Lamberti C, Mor-Avi V. Volumetric quantification of global and regional left ventricular function from realtime three-dimensional echocardiographic images. Circulation 2005;112:1161-70

19. Clopper CJ, Pearson ES. The use of confidence or fiducial limits illustrated in the case of the binomial. Biometrika 1934;26:404-13

20. Hahn GJ, Meeker WQ. Statistical Intervals: A Guide for Practitioners. New York, NY: Wiley, 1991:82-4, 100-5

21. Morey TE, Gravenstein N, Rice MJ. Let's think clinically instead of mathematically about device accuracy. Anesth Analg 2011;113:89-91

22. Cuzick J. A Wilcoxon-type test for trend. Stat Med 1985;4:87-90

23. Bland JM, Altman DG. Statistical methods for assessing agreement between two methods of clinical measurement. Lancet 1986;1:307-10

24. Hoole SP, Boyd J, Ninios V, Parameshwar J, Rusk RA. Measurement of cardiac output by real-time 3D echocardiography in patients undergoing assessment for cardiac transplantation. Eur J Echocardiogr 2008;9:334-7

25. Culp WC Jr, Ball TR, Burnett CJ. Validation and feasibility of intraoperative three-dimensional transesophageal echocardiographic cardiac output. Anesth Analg 2007;105:1219-23, table of contents

26. Corsi C, Lang RM, Veronesi F, Weinert L, Caiani EG, MacEneaney P, Lamberti C, Mor-Avi V. Volumetric quantification of global and regional left ventricular function from realtime three-dimensional echocardiographic images. Circulation 2005;112:1161-70

27. Schmidlin D, Bettex D, Bernard E, Germann R, Tornic M, Jenni $\mathrm{R}$, Schmid ER. Transoesophageal echocardiography in cardiac and vascular surgery: implications and observer variability. $\mathrm{Br}$ J Anaesth 2001;86:497-505

28. Corsi C, Coon P, Goonewardena S, Weinert L, Sugeng L, Polonsky TS, Veronesi F, Caiani EG, Lamberti C, Bardo D, Lang RM, Mor-Avi V. Quantification of regional left ventricular wall motion from real-time 3-dimensional echocardiography in patients with poor acoustic windows: effects of contrast enhancement tested against cardiac magnetic resonance. J Am Soc Echocardiogr 2006;19:886-93

29. Sapin PM, Schröder KM, Gopal AS, Smith MD, DeMaria AN, King DL. Comparison of two- and three-dimensional echocardiography with cineventriculography for measurement of left ventricular volume in patients. J Am Coll Cardiol 1994;24:1054-63 